Volume: 2, Issue: 1, January-March 2017, Pages: 156, DOI: http://dx.doi.org/10.19082/ah156

\title{
APPLICATIONS OF VIRTUAL SIMULATION IN MEDICAL EDUCATION
}

\author{
Mohammad Hosseini-ravandi ${ }^{1}$, Hesam Karim ${ }^{1}$, Elham Mousavinasab ${ }^{1}$, Ahmad Naserpour ${ }^{1}$, Reza Safdari ${ }^{2}$
}

1: PhD Student of Medical Informatics, Department of Health Information Management, School of Allied Sciences, Tehran University of Medical Sciences (TUMS), Tehran, Iran.

2: Professor in Health Information Management, Department of Health Information Management, School of Allied Sciences, Tehran University of Medical Sciences (TUMS), Tehran, Iran. (Corresponding Author)

Correspondence:

Tel: +98.9374968765, E-mail: hosseiniravandi.m@gmail.com

\section{TYPE OF ARTICLE: CONFERENCE ABSTRACT}

\begin{abstract}
Introduction: In recent years, due to the growth of IT technologies, the ways of acquisition and dissemination of information as well as the methods of learning has changed. Simulation-based learning is one of these methods. Simulation-based medical education, especially for teaching medical students is increasing every day. Simulations in medical education have various types and can be physical or virtual. The aim of this study is to review virtual simulations and their role in medical education, which is generally $3 \mathrm{D}$ content in different formats such as web applications, mobile apps, computer-based programs or games, and virtual reality.

Methods: We searched Google Scholar, PubMed, and Scopus databases to retrieve original or review articles in English. Our search strategy was (teaching OR training OR education OR e-learning) and (medical OR medicine) AND (simulation OR visualization OR 3D) in the title or the abstract of articles that were published during the recent 10 years (2007-2016).

Results: Due to creating connection between virtual and reality, virtual simulation can be an appropriate way to delivering medical content. The applications of virtual simulation in medical education can be divided into four main categories, including: 1) communication skills learning (e.g., how clinicians communicate with patients, how to do genetic counseling, communication and collaboration among medical staff, etc.); 2) treatment and clinical skills learning (e.g., learning of chronic diseases management and treatment, medication errors, etc.); 3 ) management skills training (e.g., maintaining patient safety, playing a role in the simulated management scenarios, management of a ward or operating room by simulate a health care environment to manage and reduce costs by health care professionals, etc.); and 4) practical and technical skills learning (e.g., learning how to perform a surgical procedure, practical skills learning, etc.).
\end{abstract}

Conclusion: Reviewed studies showed that virtual-simulation-based learning, in addition to creating flexibility and accessibility in delivering educational contents, can increase medical students' learning, knowledge, motivation, and self-efficacy. Although the effectiveness of virtual-simulation-based medical education is dependent on the user's previous knowledge, its various types can help to better understand medical educational content. However, more studies are needed to assess whether simulation training improves patient-related outcomes.

KEYWORDS: Virtual reality, Virtual simulation, Medical education, Medical learning, E-Learning

\footnotetext{
Abstracts of First National Congress of Medical Informatics, Mashhad, Iran, February 2017

(C) 2017 The Authors. This is an open access article under the terms of the Creative Commons Attribution-NonCommercialNoDerivs License, which permits use and distribution in any medium, provided the original work is properly cited, the use is non-commercial and no modifications or adaptations are made.
} 